\title{
Physical disaggregation of numerical model rainfall
}

\author{
Neil I. Fox and Christopher G. Collier \\ Telford Institute of Environmental Systems, School of Environment and Life Sciences, Peel Building, University of Salford, Salford M5 4WT \\ email for corresponding author: n.i.fox@salford.ac.uk
}

\begin{abstract}
A methodology is presented for the disaggregation of numerical model fields of convective rainfall using a physically based procedure. The scheme uses surface sensible heat flux values derived from high-resolution multichannel satellite radiometer observations. The sensible heat flux values initialise a simple convective model to calculate the convective disaggregation parameter (CDP), which is theoretically proportional to the convective rainfall rate. The CDP diagnostic parameter can be derived as a one-time field if the surface characteristics are invariant, as a seasonal value may be, or it may be evaluated on a case by case basis. Once found, the CDP can be used to disaggregate numerical weather prediction (NWP) convective rainfall fields for as far ahead as such fields are produced.
\end{abstract}

\section{Introduction}

Global numerical weather prediction (NWP) schemes work on grid scales of the order of $50 \mathrm{~km}$. In contrast, convective rainfall systems have dimensions of the order of a few kilometres; hence their representation within NWP models is coarse, with areas of high rainfall rates 'smeared' over hundreds of square kilometres. This effect has implications for the accurate distribution of latent heat fluxes within the numerical model and for flood forecasting, particularly in small catchments. For NWP rainfall products to be useful in water resource management, for example, over a period of days, these fields require disaggregation. Mesoscale models with grid sizes of the order of $10 \mathrm{~km}$ continue to resolve cloud scale processes poorly; at the present time, they have forecast periods of 48 hours at most. Even large eddy simulation research models which may eventually become operational for grid lengths of the order of $1 \mathrm{~km}$ (e.g. Hobson et al., 1999) are unlikely to resolve convection with enough detail to provide spatially and temporally accurate rainfall predictions for small river catchments.

Previous proposals to disaggregate model convective rainfall have used statistical methods to distribute the rainfall within a numerical model grid square (Wheater et al., 1999; Eagleson, 1984). Whilst such approaches reproduce latent heat flux patterns which reduce errors in subsequent model fields, the physical justification for positioning the rain in this way is dependent upon the mean statistical characteristics of rainfall rather than the dominant physical processes at the time of model initialisation (Chandler et al., 2000). This paper presents an alternative methodology whereby the disaggregation is based upon the physical characteristics of the surface that may influence the initiation of convection. In particular, a field of surface sensible heat flux (SHF), derived from satellite observations, is used as a lower boundary to a simple convective model of the atmospheric boundary layer (ABL). From this model, a parameter called the convective disaggregation parameter (CDP) is calculated. Theoretically, this parameter is proportional to the convective rainfall rate and can therefore be used to disaggregate the NWP model convective rainfall field.

\section{Model}

The assumption made throughout this work is that one can derive a parameter that is proportional to the convective rainfall rate at a resolution of the same order as convective storm initiation processes. The approach taken is to develop a simple model which leads to a robust diagnostic parameter. The simplicity of the scheme involves the application of a number of assumptions regarding the nature of convective air parcel ascent. The overriding assumption is that the NWP model uses detailed physics in its calculation of areal average convective rainfall and this information is retained in a disaggregated rainfall product. Therefore, the authors are justified in simplifying the convective model to obtain a physical disaggregating parameter.

The starting point for the development of such a parameter is a simple equation for rainfall rate (Wiesner, 1970): 


$$
R=\int_{z}^{z_{T}} \rho(z) w(z) \frac{\delta \chi_{s}}{\delta z} d z
$$

In this equation $R$ is the rain rate generated by the convective system in $\mathrm{mm} \mathrm{h}^{-1}$. The factors that contribute to the calculation of $R$ are the density of air $(\rho)$, the saturated mixing ratio $\left(\chi_{8}\right)$ and the vertical velocity of the ascending air parcel $(w)$. The integration takes place between the condensation level and the tropopause. In the model used for this work, the condensation level is assumed to be at the height $(Z)$ of the top of the atmospheric boundary layer (ABL). Condensation is assumed to reach the height of the tropopause $\left(Z_{\mathrm{T}}\right)$ but not penetrate any higher. As the three components of the integration have much greater values at the base of the cloud than at its top, one can approximate equation 1 by (Wiesner, 1970):

$$
R \approx \rho_{\mathrm{z}} \chi_{s z} w_{z}
$$

In Eqn. 2, all three components on the right hand side are taken as the values at the top of the ABL. $\chi_{\mathrm{s}}$ is the ratio of water present in $\mathrm{kg}$ per $\mathrm{kg}$ of air. Since $\chi_{\mathrm{s}}$ changes slowly when the atmosphere is near saturation (as it is within precipitating systems), it is assumed that this quantity is constant throughout the $\mathrm{ABL}$. To derive a parameter proportional to $R$, the height of the top of the $\mathrm{ABL}, Z$, must first be calculated. In what follows, it is assumed that airmass convection is the primary precipitation generating mechanism.

The convective ABL model used is that of a simple slab of uniform potential temperature (Carson, 1973) and the height of the top of this $\mathrm{ABL}(Z)$ is found from the surface SHF $\left(H_{s}\right)$ using:

$$
\int_{t_{t}}^{t_{2}} H_{\mathrm{s}} d t=\rho C_{\mathrm{p}} Z \Delta \theta-\rho C_{\mathrm{p}} \Delta \theta \Delta Z
$$

where $C_{p}$ is the specific heat capacity of air at constant pressure $(1004 \mathrm{Jkg}-1 \mathrm{~K}-1), \rho$ is the density of air and $\Delta Z$ is the depth of the ABL cap, which is assumed to be much less than the depth of a well-developed ABL. Hence, Eqn. 3 can be simplified to:

$$
\mathrm{Z}=\frac{\int_{\mathrm{t}_{1}}^{\mathrm{t}_{2}} H_{\mathrm{s}} d t}{\rho C_{\mathrm{p}} \Delta \theta}
$$

The integration of $H_{\mathrm{s}}$ takes place over the course of the day as the ABL develops. It is assumed to have a semisinusoidal variation between $t_{l},-18000 \mathrm{~s}$ relative to the local maximum in sensible heat, $H_{\mathrm{sm}}$, at 1400 hours, and $t_{2}$, +18000 s (after Oke, 1992). The daily maximum SHF is therefore:

$$
H_{\mathrm{sm}}=H_{\mathrm{s}} / \cos \left(2 \pi f\left(t_{\mathrm{m}}-t_{\mathrm{p}}\right)\right)
$$

where $f$ is the frequency of the heating variation $(1 / 72000 \mathrm{~s})$ and $t_{\mathrm{m}}$ and $t_{\mathrm{p}}$ are the time of maximum SHF and the time of the satellite pass from which $H_{\mathrm{s}}$ is derived, respectively.

The height of the ABL at the end of the sensible heating cycle is then found to be:

$$
\mathrm{Z}=\frac{\int_{\mathrm{t}_{1}}^{\mathrm{t}_{2} H_{\mathrm{s}} d t}}{\rho C_{\mathrm{p}} \Delta \theta}=\frac{H_{\mathrm{sm}}}{\pi f \rho C_{\rho} \cdot \Delta \theta}
$$

In Eqn. 6, $\Delta \theta$ is the potential temperature step at the top of the ABL which has been taken by Culf (1993) as being typically $2 \mathrm{~K}$.

$H_{\mathrm{sm}}$, found from $H_{\mathrm{s}}$, is now assumed to be the only locally varying parameter affecting the height of the $A B L$ and this is derived from satellite observations as described later.

If a small perturbation in the temperature of a parcel of air initially at the surface initiates convection within the $A B L$ which has a constant potential temperature $(\theta)$, then a buoyant force will be exerted upon that air parcel. If the temperature of the parcel at a height $z$ (in $\mathrm{km}$ ) is $T_{z}$ then, if it is unsaturated, it can be assumed to cool with the adiabatic lapse rate $\left(9.8 \mathrm{~K}^{\mathrm{km}} \mathrm{km}^{-1}\right)$ such that:

$$
T_{\mathrm{z}}=T_{\mathrm{a}}-9.8 z
$$

where $T_{\mathrm{a}}$ is the air temperature near the Earth's surface (screen temperature) and $z$ is the height above the surface in $\mathrm{km}$. By contrast, if the $\mathrm{ABL}$ has a constant $\theta$, then the environmental temperature $T_{E}$ is given by:

$$
T_{E}=\exp \left(\left(\frac{z}{H}\right)^{R_{d} / C_{P}}\right) T_{a}=T_{a} \exp (0.041 z)
$$

as $R_{\mathrm{d}}$ is the gas constant for dry air $\left(287 \mathrm{Jkg}^{-1} \mathrm{~K}^{-1}\right)$ and $H$ is the atmospheric scale height which is commonly approximated to $7 \mathrm{~km}$ (e.g. Wallace and Hobbs, 1977). The major unknown in these equations is now $T_{\mathrm{a}}$, which can be found from satellite observations (e.g. Price, 1989; Fox et al., 2000) or from the NWP model used.

The vertical velocity of the ascending parcel of air at the top of the ABL during convection is given by:

$$
\frac{w_{z}^{2}}{2}=g \int_{0}^{z} \frac{T_{z}-T_{E}}{T_{E}} d z
$$

Substituting the expressions for $T_{\mathrm{z}}$ and $T_{E}$ from Eqns. 7 and 8:

$$
w_{z}^{2}=\frac{2 g}{0.041 T_{a}}\left[\left(T_{a}-\frac{(0.041 Z-1)}{0.041}\right) e^{0.0412}\right]
$$

Equation 10 represents a formulation for the vertical velocity of a convective air parcel that is characterised by two spatial variables, $H_{\mathrm{s}}$ and $T_{\mathrm{a}}$, both of which can be derived using remote sensing. 
The CDP $(\varphi)$ is defined as the vertical velocity at the top of the boundary layer $\left(w_{z}\right)$ multiplied by the exponential reduction of $\rho$ to that height (cf. Eqn. 2):

$$
\varphi=w_{z} \exp (-Z / H)
$$

to normalise the value of CDP, so that the sum of the rainfall in the sub-grid square elements equals the grid square total. In this case the sub-grid square elements are defined by the pixels of the AVHRR satellite imagery used to initiate the model. The mean CDP would be equal to:

$$
\bar{\varphi}=\frac{1}{N} \sum_{i=1}^{N} \varphi_{i}
$$

where $\varphi_{\mathrm{i}}$, the CDP calculated for the $i$ th pixel, is summed over all values of $C D P$, derived from satellite data, within the numerical model grid square. Normalised pixel values of $\operatorname{CDP}\left(\varphi_{\mathrm{in}}\right)$ are then given by:

$$
\varphi_{\text {in }}=\frac{\varphi_{i}}{\bar{\varphi}}
$$

Convective rainfall amount in that pixel $\left(R_{\mathrm{ci}}\right)$ is:

$$
R_{\mathrm{ci}}=\varphi_{\mathrm{in}} R_{\mathrm{c}}
$$

Each pixel then has an associated potential convective rainfall total based on the physical measurement of the SHF at the land surface within that pixel.

\section{Model initialisation}

To use the scheme, one requires values of surface SHF and air temperature. The model is initialised using values of SHF found from remotely sensed satellite data (Fox et al., 2000). If surface characteristics do not vary significantly, one can derive one-time CDP values (or average CDP over a representative sample of days) and use them for each disaggregation procedure in that area. Alternatively, one could find seasonal values of CDP to take account of variations in the state of the vegetation.

The concept of using climatological values of CDP is adequate for larger scale model adjustment of latent heat flux fields. However, for applications of rainfall disaggregation such as flood forecasting, one requires more flexible initialisation (Fox and Collier, 2000). The major variability in surface SHF is due to soil moisture content levels that control evapotranspiration in vegetated areas. In this case, future rainfall patterns may be controlled by antecedent precipitation which reduces sensible heating by increasing evapotranspiration (Taylor, 2000). This will produce variations in the spatial contrasts of SHF if there is localised (convective) rainfall which wets one area of the grid square more than another.

Therefore, in regions where soil moisture content is variable, to use the physical disaggregation technique for flood forecasting, one needs to evaluate directly the soil moisture availability (e.g. Njoku and Entekhabi, 1996, Fox et al., 2000) or the antecedent precipitation index (Arkin and Meisner, 1987).

\section{Case study}

An example of a disaggregated field is presented in Fig.1 from the northwest of England for 1800Z on 22nd July 1996. This is a disaggregation of a single grid square of the Met Office Unified Model (see, for example, Wilson and Ballard, 1999) convective rainfall run at $0000 Z$ on 20th July 1996. The disaggregation is achieved using a field of SHF derived from NOAA AVHRR data collected at $1300 \mathrm{Z}$ on 20 th July 1996. No disaggregation is presented over the sea surface as it exhibits such uniformity that convective initiation by variable SHF will not occur over open sea. Some pixels have no associated value (shown as white) because the surface was too complex to resolve reasonable values of SHF by the remote sensing methodology. This occurs in urban centres and so a bias may be introduced by neglecting these particular areas. The non-registration of a small number of pixels in urban areas leads to a bias in the normalisation scheme and improvements should be made in the remote sensing scheme to remove this problem.

The areas that produce high rainfall rates are the urban areas. This is due to the reduced evapotranspiration which leads to higher sensible heat fluxes in these areas. Figure 1 identifies the regions of enhanced convective initiation due to the conurbations of Liverpool (southwest of figure) and Manchester (central south). The areas where no convective initiation is forecast are those of the upland Pennine Moors and densely vegetated Rossendale Forest toward the north of the area. If one assumes that convective rainfall is produced by enhanced sensible heating alone then the pattern appears good. The figure indicates areas in which convection is preferentially strong but, to forecast rainfall location accurately, one must advect the pattern using boundary layer wind velocities from the numerical model as the winds at these levels determine the lateral motion of the parcel as it ascends between the surface and the condensation level.

Figure 2 shows the track of rain-producing cells detected by the Met Office Hameldon Hill radar between 1930 and 2200 on 22nd July 1996. These cells form in the vicinity of the south western 'hotspot' corresponding to the city of Liverpool. This area is identified in Fig. 1 as being an area where convection is preferentially initiated. The cells then 

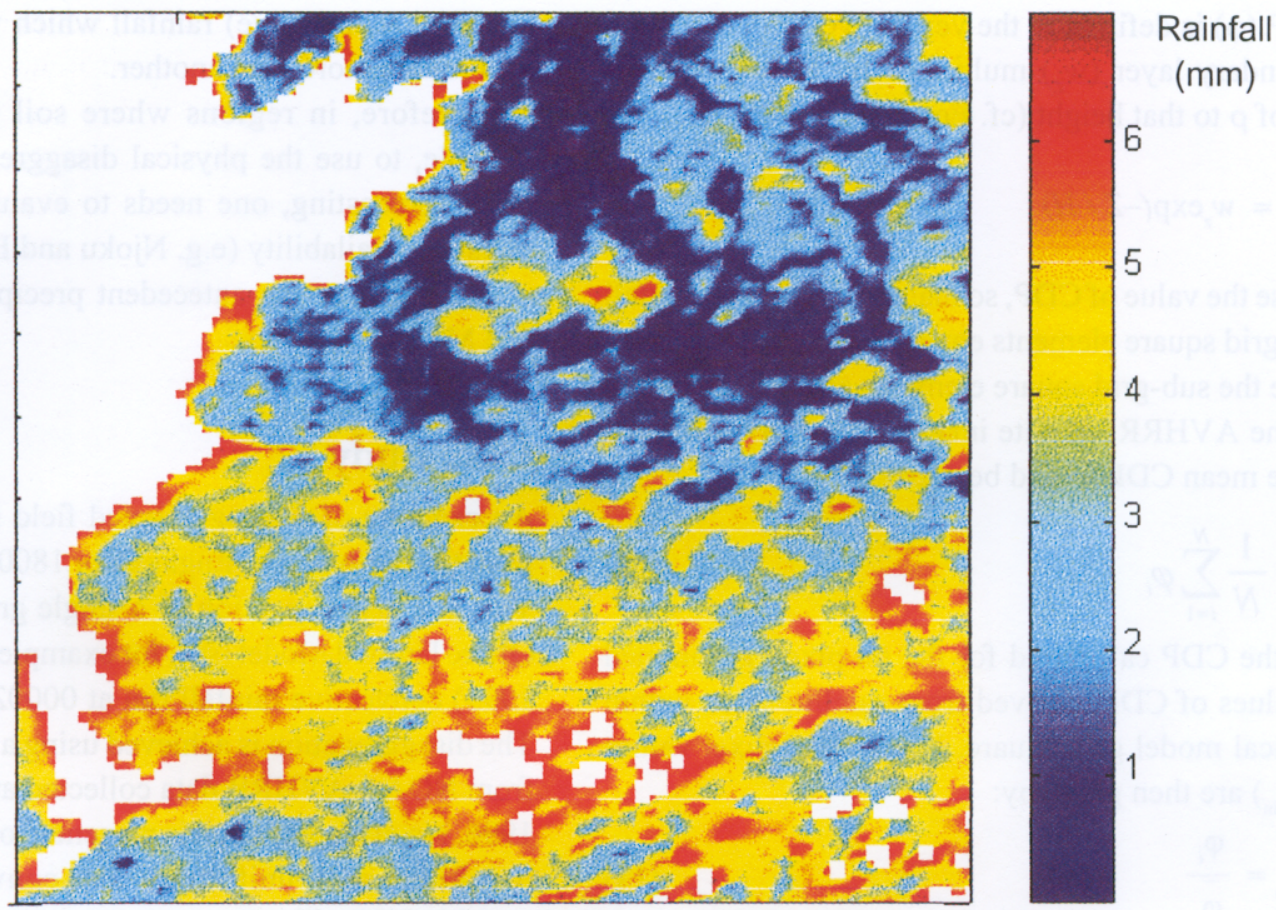

Fig. 1. Disaggregated field of convective rainfall for 3 hour period $1800 \mathrm{Z}$ to $2100 \mathrm{Z}$ on $22^{\text {nd }}$ July 1996 from UKMO Unified Model run at $0000 Z$ on $20^{\text {th }}$ July 1996 and SHF derived from NOAA AVHRR data from $1300 Z$ on $20^{\text {th }}$ July 1996

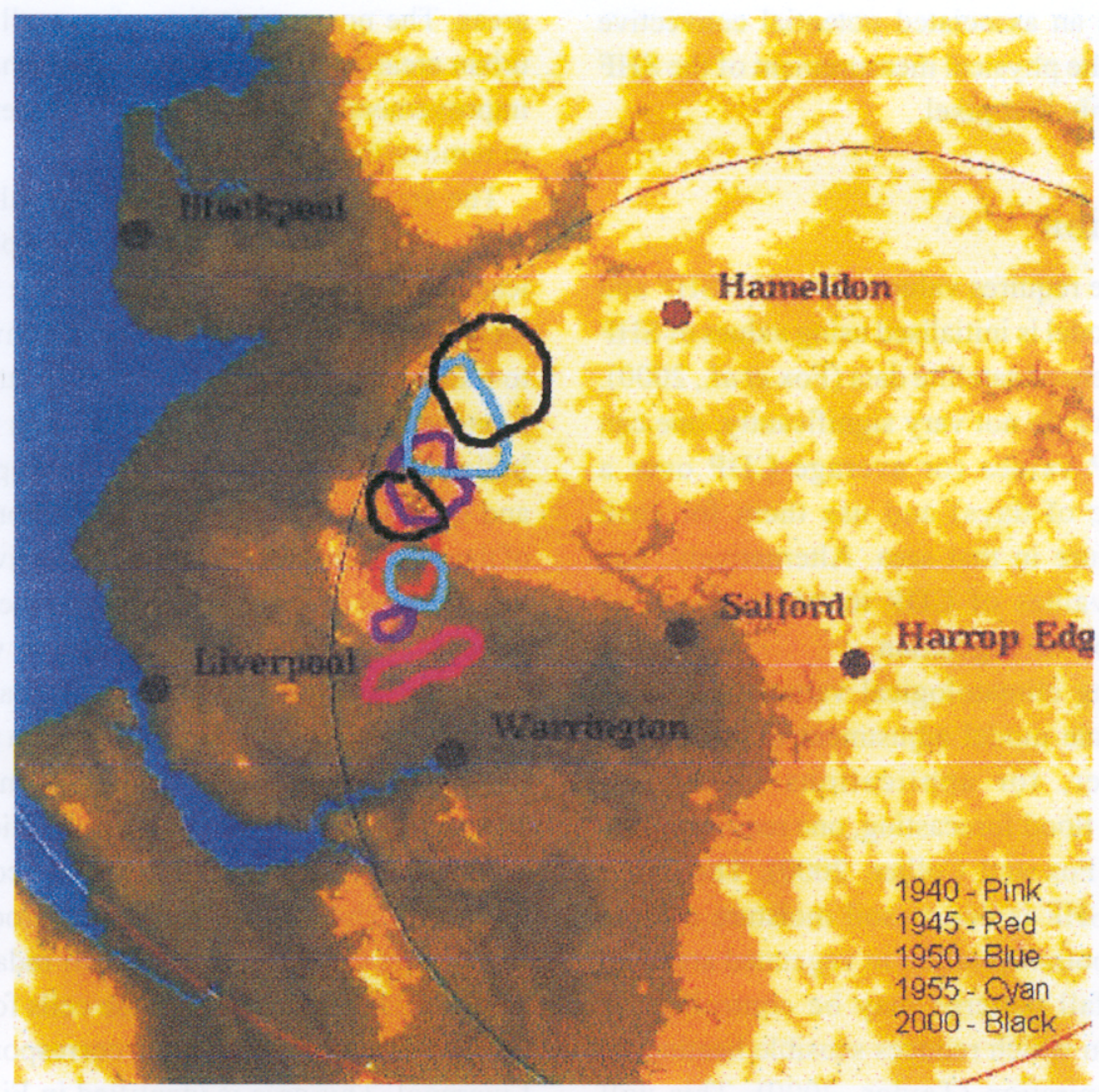

Fig. 2. Track of storm cell as observed by Hameldon Hill radar between 1930 and 2000 on $22^{\text {nd }}$ July 1996 
track north-northwest and intensify over the higher ground of the West Pennine Moors.

\section{Discussion}

The case study demonstrates the potential of the disaggregation scheme. The patterns generated by the model appear reasonable and are similar to those produced by statistical methods. In this case, however, the patterns are based on the underlying physical characteristics of the surface.

The surface features which are clearly of greatest importance in the region where the study took place are urban in character. The model suggests increased convection over these areas. However, actual rainfall will occur downwind of the point of convective initiation. Therefore, one needs to advect the disaggregated precipitation pattern with the model windfields.

The disaggregation scheme has been developed to be as simple as possible, whilst grounded in a physical understanding of convective initiation. There are, therefore, numerous limitations and improvements that can be made. The limitations revolve around the necessity to recognise that the disaggregation process assumes that the convection is generated by SHF variations. Hence, if the convection is produced by orographic forcing or boundary layer convergence, then the position of cells will be independent of surface SHF extremes.

The use of a parameter (CDP) that is normalised reduces the sensitivity of the scheme to the values chosen for constants such as the temperature $\operatorname{step}(\Delta \theta)$ and the environmental lapse rate. The model is sensitive to differences in sub-grid element values of $\varphi$ rather than its absolute value, even though this can be interpreted physically in terms of the vertical velocity. This does not imply that the scheme is insensitive to these parameters or to the exact formulations used; the variations in the field caused by altering the parameterisations must be examined in the context of a larger study of the scheme.

In the case study, considerable convective cell development resulted from the advection of ascending air parcels into areas of raised ground. This indicates the importance of the addition to the scheme of the component of vertical wind velocity produced by orographic uplift. Vertical velocities caused by orography are often of greater magnitude than those caused by sensible heating, especially outside of summertime conditions. Given access to numerical model wind fields and a digital elevation map of the area of the same resolution as the SHF field, one could produce this component of vertical motion.

The convective model is one-dimensional and does not allow for interaction between air motions in adjacent areas.
In real convective systems, extremely vigorous ascent is generated by circulations produced by differences in convective driving forces. Therefore, it seems reasonable that the disaggregation should be based, not on the absolute SHF alone, but upon gradients in the field of SHF.

The current version of the model would, no doubt, best be applied in areas of less complex landscape variation. Interpretation of the fields produced by the scheme would be easier in regions lacking large urban developments and less marked orographic features. If the model were examined using data from a more tropical climate where rainfall is generally more convectively forced (rather than dynamically forced), greater clarity of results could be achieved. It is hoped that further development of the model can be coupled to tests in such areas.

\section{Acknowledgements}

The authors thank Peter Panagi and Ed Dicks of the JCMM for their help with the UM data which was acquired through the UWERN network. The AVHRR data was provided through the NERC receiving station at Dundee.

\section{References}

Arkin, P.A. and Meisner, B.N., 1987. The relationship between large-scale convective rainfall and cold cloud over the western hemisphere during 1982-84. Mon. Weather Rev., 115, 51-74.

Carson, D., 1973. The development of a dry inversion-capped convectively unstable boundary layer. Quart. J. Roy. Meteorol. Soc., 99, 450-467.

Chandler, R.E., Mackay, N.G., Wheater, H.S. and Onof, C., 2000. Bayesian image analysis and the disaggregation of rainfall. $J$. Atmos. Ocean Tech., 17, 641-650.

Culf, A.D., 1993. The potential for estimating regional sensible heat flux from convective boundary layer growth. J. Hydrol., 146, $235-244$.

Eagleson, P.S., 1984. The distribution of catchment coverage by stationary storms. Water Resour. Res., 20, 581-590.

Fox, N.I. and Collier, C.G., 2000. Estimating medium-range catchment flood potential. J. Hydrol., 237, 1-16.

Fox N.I., Saich P. and Collier, C.G., 2000. Estimating the surface water and radiation balance in an upland area from space. Int. J. Remote Sens., 21, 2985-3002.

Hobson, J.M., Wood, N. and Brown, A.R., 1999. Large-eddy simulations of neutrally stratified flow over surfaces with spatially varying roughness length. Quart. J. Roy. Meteorol. Soc., 125, 1937-1958.

Njoku, E.G. and Entekhabi, D., 1996. Passive remote sensing of soil moisture. J. Hydrol., 184, 101-129.

Oke, T.R., 1987. Boundary Layer Climates. 2nd edition. Routledge, London. 435pp.

Price, J.C., 1989. Quantitative aspects of remote sensing in the thermal infrared. In Theory and applications of optical remote sensing, G. Asrar (Ed.), Wiley, New York, 578-602.

Taylor, C.M., 2000. The influence of antecedent rainfall on Sahelian surface evaporation. Hydrol. Process., 14, 1245-1259.

Wallace, J.M. and Hobbs, P.V., 1977. Atmospheric Science: An introductory survey. Academic Press, London. 467pp. 